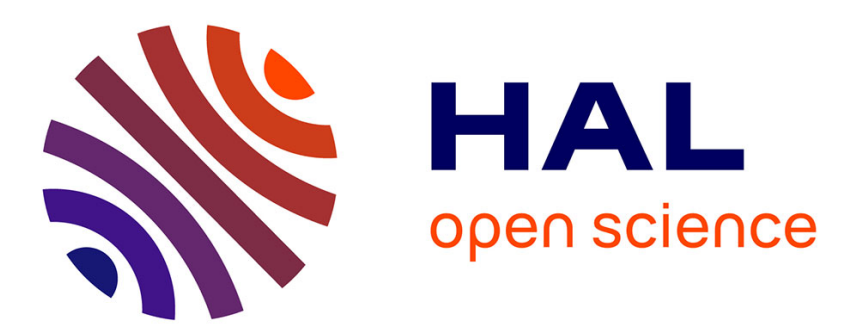

\title{
Practical performances of high-speed measurement of gear Transmission Error or torsional vibrations with optical encoders
}

Didier Rémond

\section{- To cite this version:}

Didier Rémond. Practical performances of high-speed measurement of gear Transmission Error or torsional vibrations with optical encoders. Measurement Science and Technology, 1998, 9 (3), pp.347353. 10.1088/0957-0233/9/3/006 . hal-00692560

\section{HAL Id: hal-00692560 https://hal.science/hal-00692560}

Submitted on 5 Nov 2018

HAL is a multi-disciplinary open access archive for the deposit and dissemination of scientific research documents, whether they are published or not. The documents may come from teaching and research institutions in France or abroad, or from public or private research centers.
L'archive ouverte pluridisciplinaire $\mathbf{H A L}$, est destinée au dépôt et à la diffusion de documents scientifiques de niveau recherche, publiés ou non, émanant des établissements d'enseignement et de recherche français ou étrangers, des laboratoires publics ou privés. 


\title{
Practical performances of high speed measurement of gear transmission error or torsional vibrations
}

\section{with optical encoders.}

\author{
Didier REMOND \\ Laboratoire Conception et Analyse des Systèmes Mécaniques \\ I.N.S.A de Lyon, Bâtiment 113 \\ 20, Avenue Albert EINSTEIN \\ 69621 VILLEURBANNE Cedex, FRANCE \\ E-mail : didi@casm.insa-lyon.fr
}

\begin{abstract}
We evaluated in this paper an improved technique to measure Gear Transmission Error (GTE) at high speed, by using low pulse per revolution optical encoders. The originality of this technique lies on the fact that highly precise, completely digital measurements of torsional vibration or Transmission Error (TE) at high speed are achievable by the use of low price, basic optical components. The length of encoder pulses are estimated with a high frequency timer $(100 \mathrm{MHz})$ : thus, it appears that the theoretical precision of this device only depends on the angular speed of shafts, but not on the number of pulses per revolution of the encoder. In practice, intrinsic encoder accuracy (i.e. grating or electronic signal processing
\end{abstract}


precision) directly affects precision measurements. Alternatively, the number of pulses per revolution of the encoder specifies the resolution.

We examined the possibility of calibrating encoders through a specific test rig. The determination of corrective data assigned to each gratings leads to a non significant improvement of the precision measurement. The coherence from one revolution to another does not present any significant deterministic component. The overall precision achieved is less than 0.03 second of arc on each frequency of the power spectral density.

This calibration device only gives a good assessment of eccentricity induced by mechanical mounting of optical disks on a shaft, compared with the direct measurement on grating disks. The correlation between the two measurements is less than $3 \%$ of the magnitude of the relative eccentricity.

Thus, encoder technique seems to be a cheap and easy way to implement Transmission Error measurement on real mechanical systems with high precision and sufficient reliability.

\section{Notation}

(arcsec) second of arc, (angular unit)

$\mathrm{fh}_{\mathrm{h}} \quad$ frequency of timer, in $\mathrm{Hz}$

$\mathrm{N}$ number of pulses per revolution

$\Delta \mathrm{N}_{\mathrm{h}} \quad$ number of timer impulses to cover 1 second of arc

$\Omega \quad$ mean angular speed, in rad.s ${ }^{-1}$

$\Delta \Omega \quad$ speed fluctuation, in rad.s ${ }^{-1}$

ppr pulse per revolution 
$r \quad$ radius of encoder sensor

$\Delta r \quad$ eccentricity of encoder disk

$T_{r}, T_{r+\Delta r}$ time length of one period of encoder signal for optical cell radius of $r, r+\Delta r$

$\Delta T \quad$ mean time of one period of encoder signal, in seconds

$\Delta \mathrm{T}_{\mathrm{m}} \quad$ mean time of one period of encoder signal, in number of timer impulses

$\Delta \theta_{\mathrm{i}} \quad$ angular displacement during one timer pulse, in second of arc

(tp) timer pulses (time unit)

\section{Introduction}

Concomitant attempts to understand gear noise generation and to validate numerical models of gear behaviour require the assessment of various gear transmission parameters, particularly precise measurement of gear transmission error. However, measurement of gear transmission error in real operating conditions (especially at high speed) requires very specific techniques which are not easy to implement.

The desirable precision of transmission error measurement depends on the size and accuracy of gears. For automotive gears of about $100 \mathrm{~mm}$ in diameter, a precision of about $0.25 \mu \mathrm{m}$ is equivalent to an angular step of 1 second of arc. Thus, reliable measurements of transmission error has to be below this limit to be relevant. A linked problem is the high frequency limit of electronic signals when both high speed and high precision are required. A 36000 pulses per revolution encoder provides a 3,6 MHz signal at a rotation speed of $6000 \mathrm{rpm}$, with a pulse spacing of 36 seconds of arc (defining the precision).

In order to perform a high precision - high speed measurement, the approach we have been using for five years has been improved and leads to a measurement 
set-up based on a specific acquisition board and industrial encoders. This method has been widely used, but its description and analysis remains to be fulfilled. This paper deals with the accuracy and the performances of the proposed angular measurement device. First of all, the purpose of this work is to define the entire measurement process. Then, we determined the influence of optical encoder accuracy in the acquisition process. In order to study the possibility of calibrating encoder defaults, a mechanical set-up to test optical encoders has been used. Different kinds of encoders have been used and their performances compared. Thus, the possibility to correct encoder defaults, like grating position, is examined for one encoder feature, taking into account the mechanical set-up behaviour. We conclude that there is no significant precision improvement given by the calibration of gratings on each encoder, except the eccentricity induced by disk mounting on the shafts.

\section{Phase difference measurement device.}

For some years, mechanical devices such as power transmission have been considered as the main source of excitation and noise generation. The most representative mechanical characteristic is the TE. In geared systems, TE is defined as the difference between the angular position of the wheel and the position it would have had if the transmission was perfect (infinite stiffness and perfectly conjugated teeth) in reference with pinion position [5]. Numerous works have been published on gear transmission error measurement $[1,3,4,10,11]$ using both torsional accelerometer techniques and optical encoder devices. Under similar experimental conditions, the T. E. results obtained from both techniques fit generally quite well [11]. 
From a general point of view, it is difficult to combine high precision assessments with high speed measurements when using optical encoders, as the use of an acceptable number of pulse per revolution meets with frequency limitation of the generated signals. The number of pulses specifies the resolution and has to be chosen about 5 times greater than the number of cycles of the TE harmonic of interest. Moreover, couplings or mechanical parts generate mechanical resonances at high speed, which interfere with the measurements.

In our case, an interesting way to solve these problems of speed limitations associated with optical encoder devices consists (i) in using "through shaft" encoder (Fig.4) to avoid flexible coupling behaviour for the first part, and (ii) in dissociating the angular sampling or resolution, performed by encoders, from the precision performance, obtained by using a $100 \mathrm{MHz}$ timer [1]. This time counting method allows the determination of phase difference with a low number of pulses per revolution (ppr) and seems to be reliable for phase difference estimation [14]. Furthermore, it is important to note that the gear ratio is taken into account numerically in the case of transmission error measurement. Another important point is that the two channels are operating with the same timer and the same counter, giving a robust reference to pulse timing. On the opposite, the "standard method" [10] uses "judicious" dividing process in order to compare two signals of about the same "low" frequency. But analogic PPL circuits are required to perform one or two divisions (on each shaft) which are equivalent to a division of the number of pulses per revolution (on each encoder). Moreover, the phase difference magnitude is limited by these PLL circuits, mixing digital and analogic treatments at different steps of the process. Finally, this standard method involves different process on each encoder signal, source of perturbations on phase difference measurement. 
In figure 1, the angular position of each encoder (and consequently, the position of linked gear or shaft) is obtained with high accuracy by counting timer pulses (tp) between each encoder rising edge. Then, the evolution of the angular position of each shaft can be processed and the simultaneous analysis of the two encoder signals allows the computation of the gear TE by re-sampling each angular position with linear interpolation. A simplified scheme of the working principle is presented in Figure 2. Note that the overall number of acquisition points is only limited by the amount of computer memory.

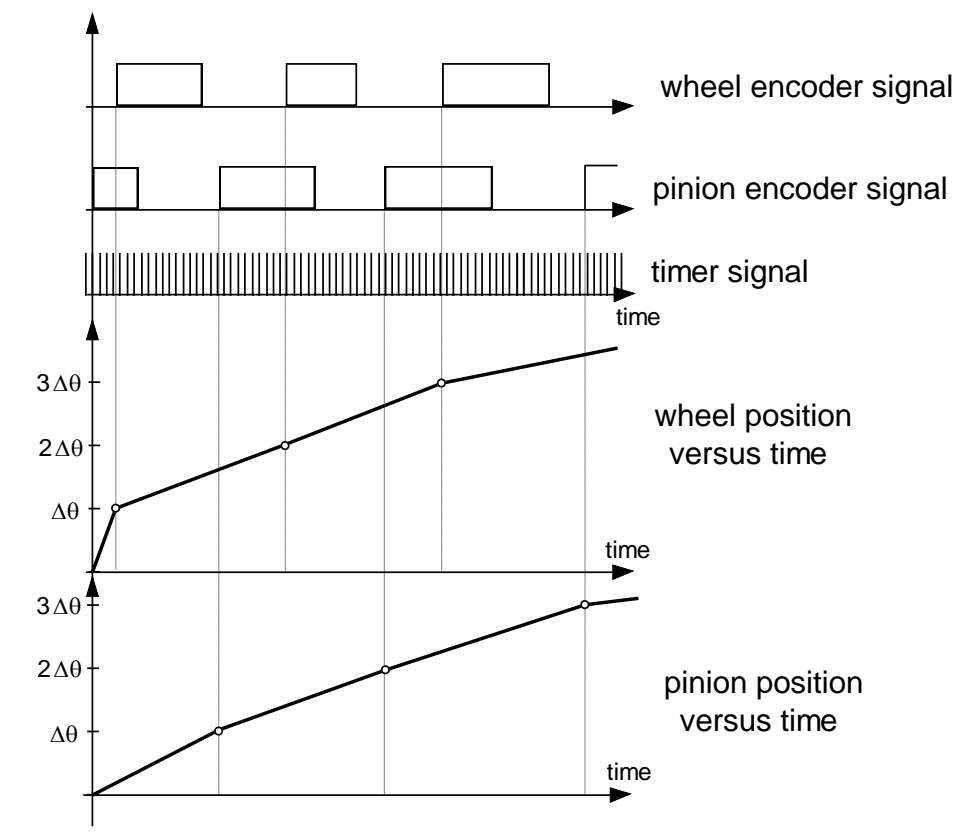

Fig. 1 : Building of angular position laws for the pinion and the wheel.

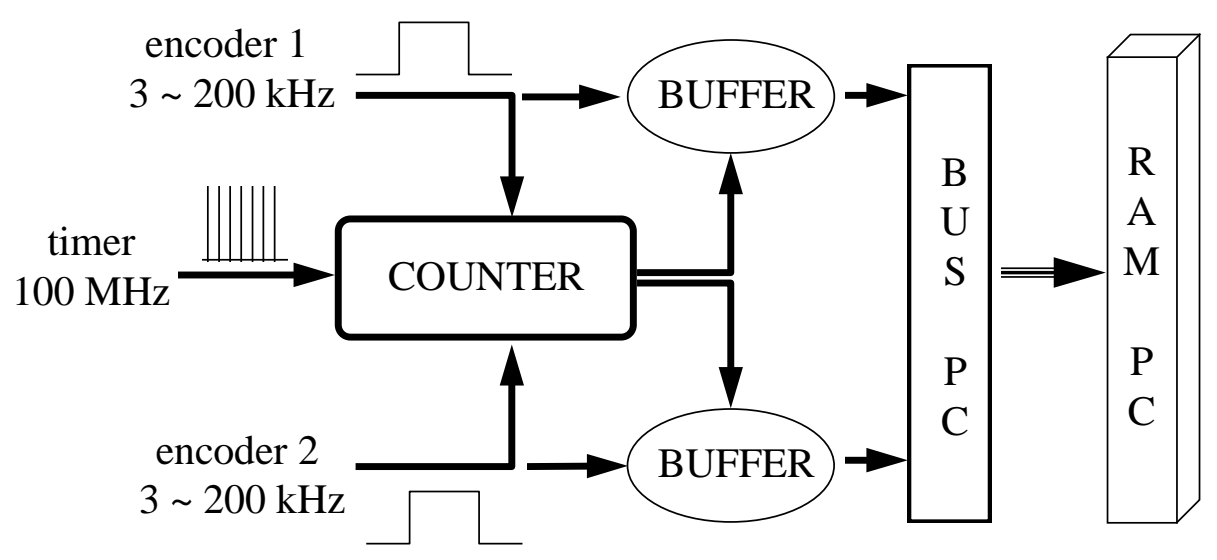


Fig. 2 : Working principle of the TE acquisition board.

In order to appreciate the level of precision given by such a device, some first indications can be given. The theoretical precision is set up by the fact that two events can be separated at least by a timer pulse. That is to say at operating speed, the possibility to have an angular precision of :

$$
\Delta \theta_{\mathrm{i}}=\frac{360 \cdot 60 \cdot 60 \cdot \Omega}{2 \pi \cdot \mathrm{f}_{\mathrm{h}}} \quad(\text { in } \operatorname{arcsec})
$$

or

$$
\Delta \mathrm{N}_{\mathrm{h}}=\frac{2 \pi \cdot \mathrm{f}_{\mathrm{h}}}{360 \cdot 60 \cdot 60 \cdot \Omega}=\frac{\mathrm{N} \cdot \Delta \mathrm{T}_{\mathrm{m}}}{360 \cdot 60 \cdot 60} \quad \text { (in tp) }
$$

For example, a rotation speed $\Omega$ of $100 \mathrm{rad}^{-1}$ (approximately $1000 \mathrm{rpm}$ ) leads to a theoretical precision $\Delta \theta_{i}=0.216$ second of arc. Moreover, these relations imply that this theoretical precision only depends on the speed and never on the encoder number of pulses per rotation. Another important assumption is that the speed has to be constant during an encoder pulse. Speed fluctuations are mixed with angular displacement since measurements are made only on the time length between pulses. So, in order to keep a precision of 1 second of arc, speed fluctuations must be less than :

$$
\frac{\Delta \Omega}{\Omega}=\frac{\mathrm{N}}{360 \cdot 60 \cdot 60+\mathrm{N}}
$$

This can be expressed in angular acceleration as :

$$
\frac{\Delta \Omega}{\Delta \mathrm{T}}=\frac{\Delta \Omega}{\Omega} \cdot \frac{\Omega}{\Delta \mathrm{T}}=\frac{\mathrm{N}^{2} \cdot \Omega^{2}}{(360 \cdot 60 \cdot 60+\mathrm{N}) \cdot 2 \pi}
$$


For the same given speed of $100 \mathrm{rd}^{\mathrm{s}} \mathrm{s}^{-1}$ and with $4096 \mathrm{ppr}$ encoder, this leads to speed fluctuation of $0.315 \%$ along the length of encoder pulse, corresponding to an

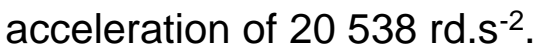

A very easy way to carry out an experiment to assess this precision consists in generating a square wave at a constant frequency by a function generator, and measuring difference from the mean value at different timer frequencies of the acquisition board on the two channels. The curve shown in the figure 3 gives results in frequency domain when tests are performed with an arbitrary intermediate frequency of $1252 \mathrm{~Hz}$ for timer frequencies of $100 \mathrm{MHz}$ (higher precision). The recorded fluctuations of the length of generated signal periods are mainly due to the performances of the generator and are white noise components, excepted for the peak located at approximately 60 cycles per second ( 0.1 in normalised frequency), corresponding to the frequency of power supply. 


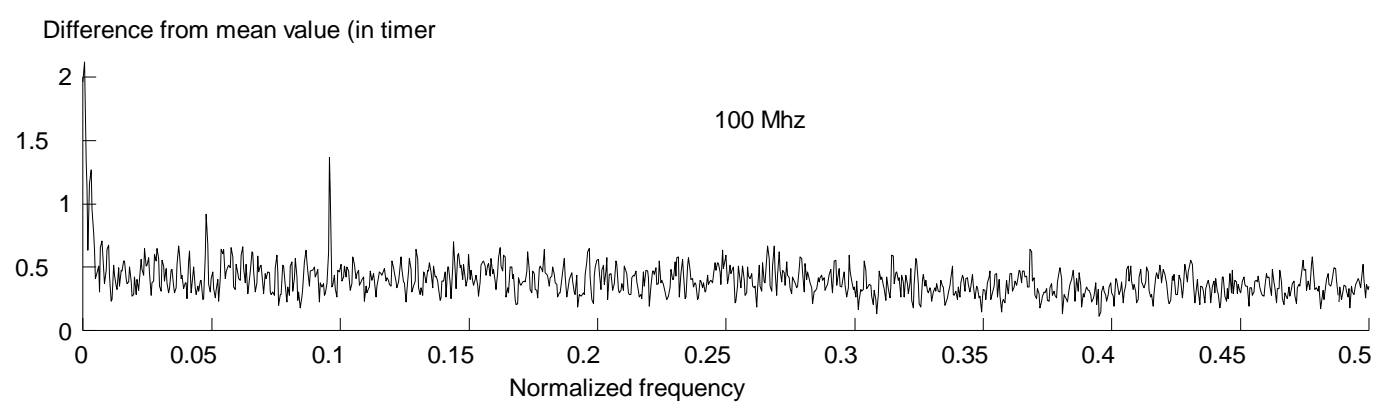

Fig. 3 : Frequency analysis of calibration signal at $1252 \mathrm{~Hz}$

$($ Clock speed $=100 \mathrm{MHz})$

Moreover, note that the two channels give the same length when records of one same signal are performed. This implies that the threshold occurs at the same moment and leads to the same time length on the two channels.

The electronic processing behaviour of the acquisition board is very satisfactory and equivalent on the two channels.

It must be added that the simulated precision is under the value of 1 timer pulse on each frequency component, and this is obtained with fluctuations induced by the function generator. Thus, the precision of the acquisition board is expected to be largely satisfactory under real operating conditions. We aim now at establishing the localisation precision of each encoder pulse and to improve the lack of precision by the determination of systematic errors allocated to each encoder grating.

\section{Encoder calibration test apparatus.}

The mechanical device, shown in figure 4 , is made of two roller bearings which support the main shaft and a heavy flywheel, in order to reduce short-term speed variations. Rotation is assumed by an accurate A.C motor through two stage of belt transmission, to give effective speed ratio and stability. Measured 
eccentricities on each pulley are less than $25 \mu \mathrm{m}$, leading to a good stability in revolution speed.
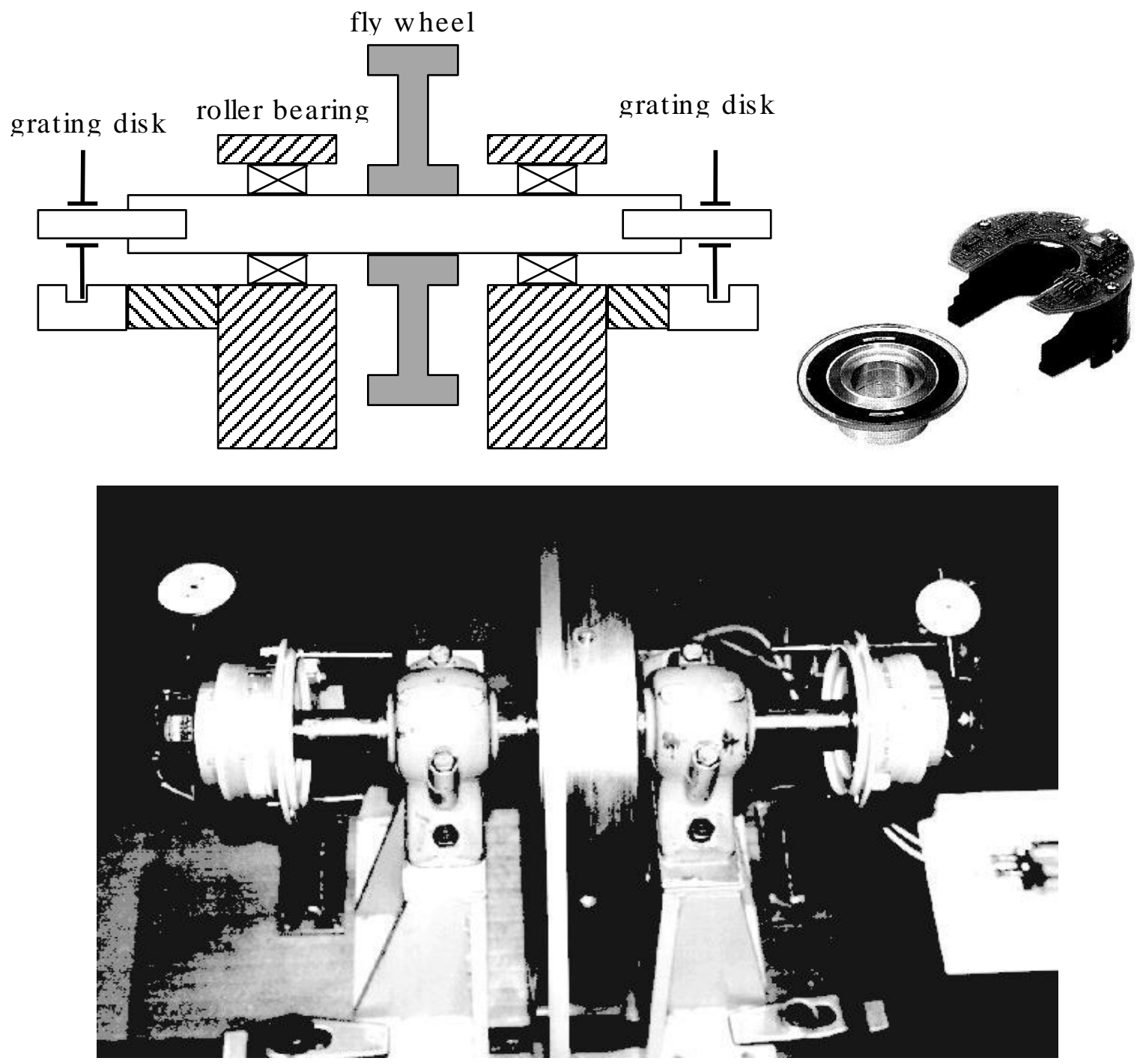

Fig. 4 : Scheme and pictures of apparatus set-up and "through shaft" encoders

Encoders are clamped symmetrically on both side of the shaft, without couplings to avoid torsional dynamic effects, eccentricity and misalignment mountings. Different kinds of encoders can be mounted on the test rig and comparisons are made with the same "through shaft" encoder as reference.

These reference encoders have a resolution of 4096 pulses per revolution (ppr), while classical encoders have two different resolutions of 2048 and $1800 \mathrm{ppr}$ with different accuracy in disk grating location. In order to keep a meaningful 
precision for calibration, rotational speed is reduced to approximately $18 \mathrm{rpm}$, given a maximum frequency for encoder signal of $1230 \mathrm{~Hz}$. In these operating conditions, theoretical precision reaches $3.810^{-3}$ arcsec.

\section{Comparison of encoders with different manufacturing accuracy.}

Different kinds of encoders have been tested on this set-up, in order to test if it is possible to detect singular defaults on pulse location, and to prevent these defaults by a calibration of encoders. Three encoders were tested, each of them having a specific level of accuracy and a different number of pulses per revolution. It is obvious that having more pulses per revolution needs to have an improved quality in gratings location and in electronic restitution of signals. The time responses of the encoders for one revolution cycle are presented in figure 5 with the mean value of each period (expressed in tp) and the standard deviation in seconds of arc, calculated on a single revolution. As mentioned before, the through shaft encoder with the greater number of pulses per revolution leads to fluctuations above 0.2 second of arc, while the classical low resolution encoders give fluctuations of about 2 seconds of arc. These first results include mechanical behaviour of the apparatus, particularly the bearing effects, motor speed fluctuations and transmission imperfections from pulleys and belts. These effects seem to be negligible when comparing the three signals, but are detectable when the precision of encoder becomes sufficient. 


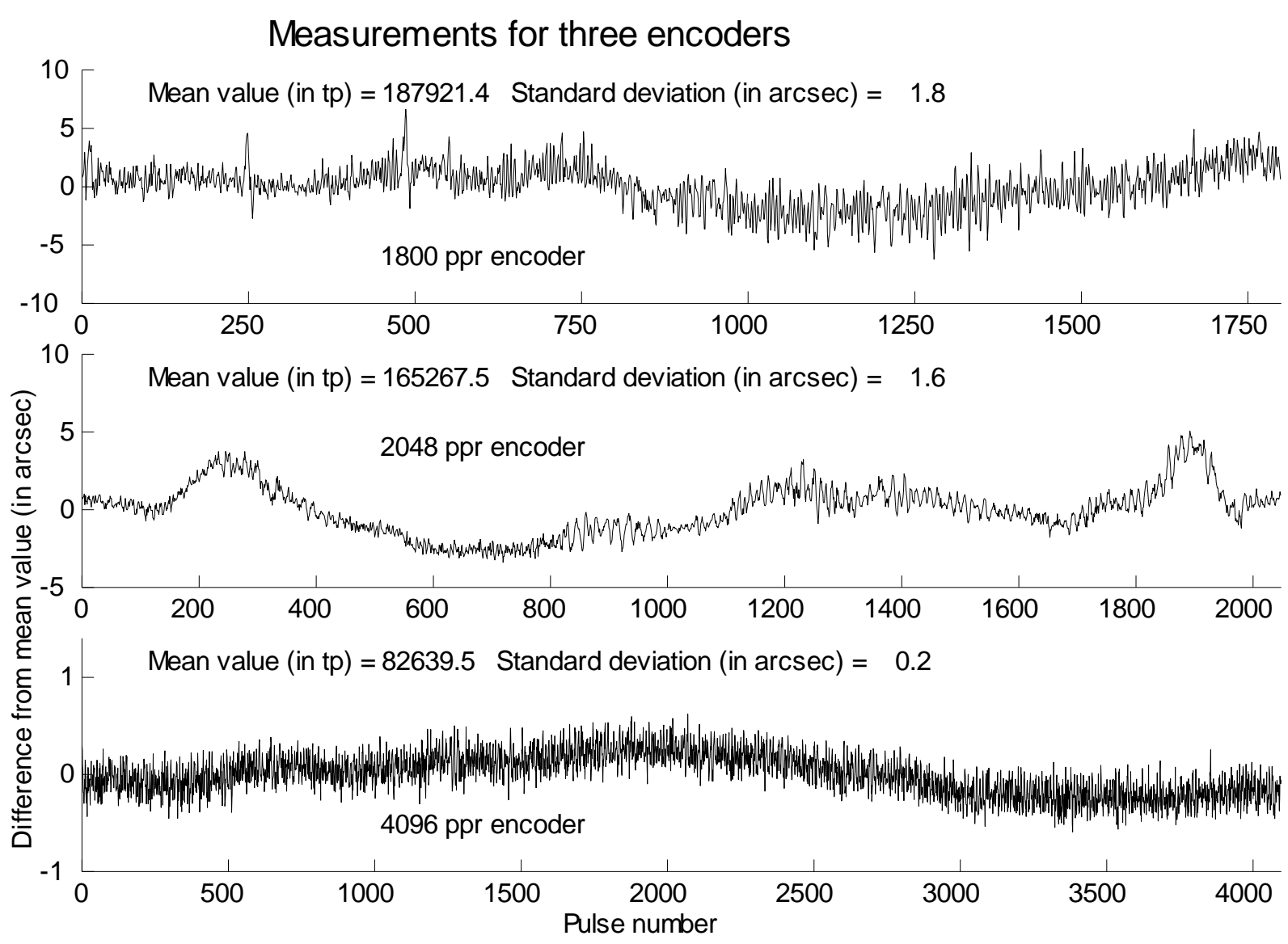

Fig. 5 : Time comparison of one revolution signal from three different encoders.

For the 4096, there is no significant deterministic component which can be attributed to a default process, in manufacturing or in operating these encoders. A spectral analysis of each signal (figure 6a and 6b) gives spectral density of these encoder signatures. It is important to note that the overall white noise component for the $4096 \mathrm{ppr}$ encoder are about 0.01 second of arc, giving such a precision for all spectral analysis in future TE measurements. Higher components at very low frequency are due to mechanical behaviour of the test bench.

\section{Corrections on $4096 \mathrm{ppr}$ encoders.}


In order to validate these results, simultaneous measurements have been performed on the two through shaft encoders, in order to eventually correct on each encoder device. The coherence function between two revolutions of the same encoder has to give information about periodic events which would take place during one revolution, in a repetitive manner, on this encoder. High coherence component should be characteristic of defaults on the encoder. But mechanical behaviour is expected to have an effect on these analysis : thus the coherence function between the two encoders should be indicative of mechanical behaviour of the apparatus. The coherence function is calculated between two encoders running simultaneously. From this curve, we detect one frequency range where coherence becomes significant, at very low frequency, corresponding to a 8 per cycle event. This can be also seen in the figures 5 and can be related to the bearing behaviour. Without giving more details, these components have the appearance of small disturbances in the running of our test apparatus. There is no other significant component which appears periodically on measurement and can be noticed for the next study concerning correlation in between revolutions.

The figure 6 gives the coherence function between two different revolutions of the same encoder. Similar results were obtained with the second encoder (data not shown). 


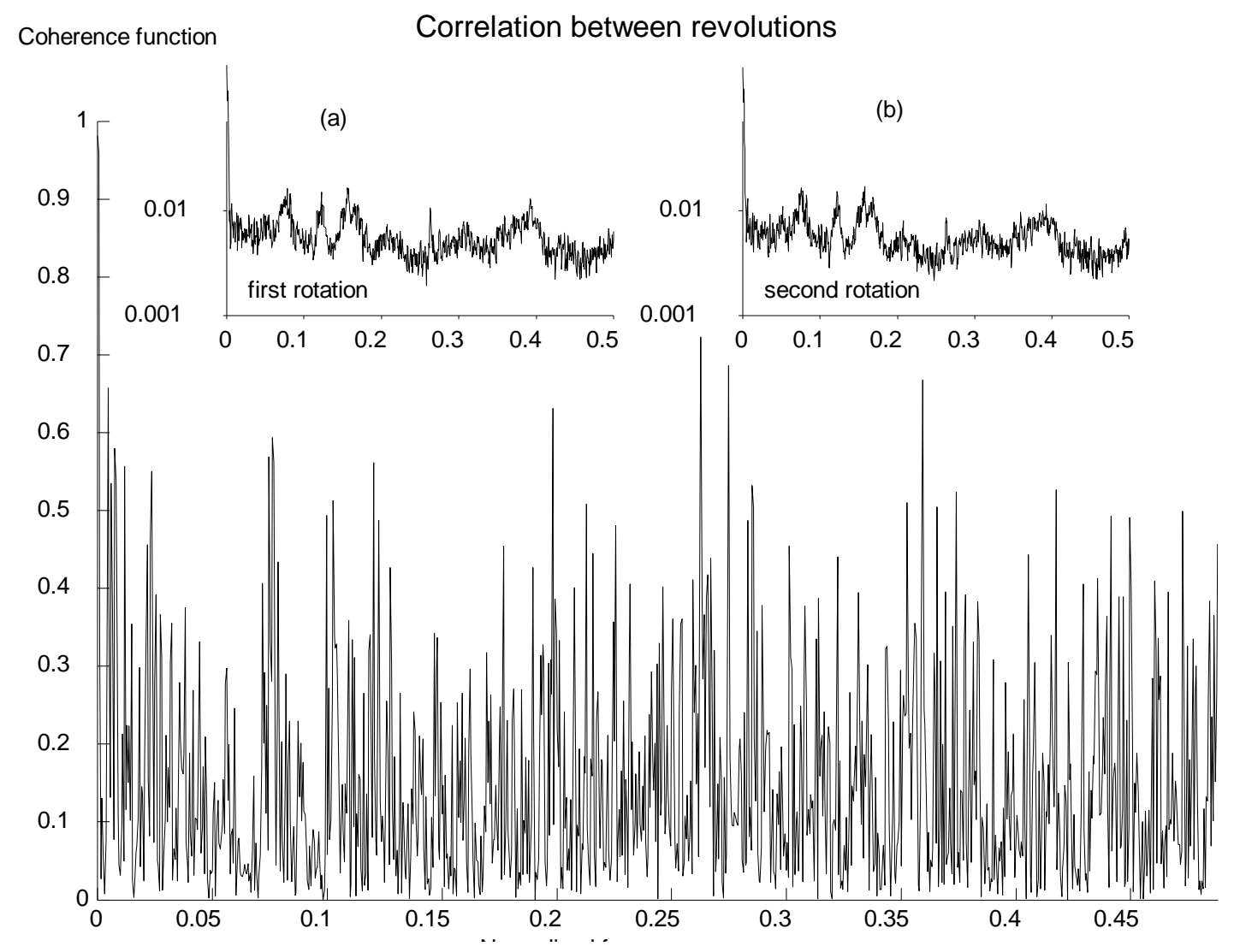

Fig. 6 : Correlation between two rotations of the same encoder :

coherence function and the two power spectral density ( $a$ and $b$ ).

For any interesting component of frequency, the coherence function is smaller than 0.7 and again, there is no periodic event which is coherent from one revolution to the other. In other words, there is no significant manifestations of manufacturing process on pulse location, which is characteristic of the encoder. Some combinations have been made from one revolution to the other, in order to extract corrections at each pulse, but they only lead to non significant modifications of fluctuations seen in the signal. That is to say, no corrective data can be calculated in order to calibrate or improve measurement made with these encoders.

The figure 6 also shows two power spectral density functions resulting from two rotations : they exhibit no significant difference (and present no component due to lightening disturbance). 
After visualisation of many measurements in time domain, there is a one per revolution component which can become important and which does not appear clearly on these curves. This is mainly due to the numerous relative positions of the different rotation axes, which introduce eccentricities. In order to be able to correct this effect, difference in position of the two encoder signals is calculated as TE measurement is performed (with a speed ratio of 1 ).

It is possible to estimate the difference in time length of pulses induced by a fluctuation of the operating radial position of encoder disk from optical sensor at a constant speed. Considering this variation of time length (essentially due to an increase of the arc length between two gratings), the difference can be expressed by :

$$
\mathrm{T}_{\mathrm{r}+\Delta \mathrm{r}}-\mathrm{T}_{\mathrm{r}}=\frac{\theta}{\Omega} \cdot \frac{\Delta \mathrm{r}}{\mathrm{r}} \quad \text { (in s) }
$$

or

$$
\mathrm{T}_{\mathrm{r}+\Delta \mathrm{r}}-\mathrm{T}_{\mathrm{r}}=\mathrm{T}_{\mathrm{m}} \cdot \frac{\Delta \mathrm{r}}{\mathrm{r}} \quad \text { (in tp) }
$$

This fluctuation of time is interpreted as a fluctuation of relative angular position of the two encoders, and then could be rewritten as :

$$
\Delta \theta=\frac{360 * 60 * 60}{\mathrm{~N}} \cdot \frac{\Delta \mathrm{r}}{\mathrm{r}} \quad(\text { in } \operatorname{arcsec})
$$

Measuring the eccentricities of the two encoder disc in reference of encoder support with dial gauges gives the curves in the figure 7 . 


\section{Relative eccentricitv}
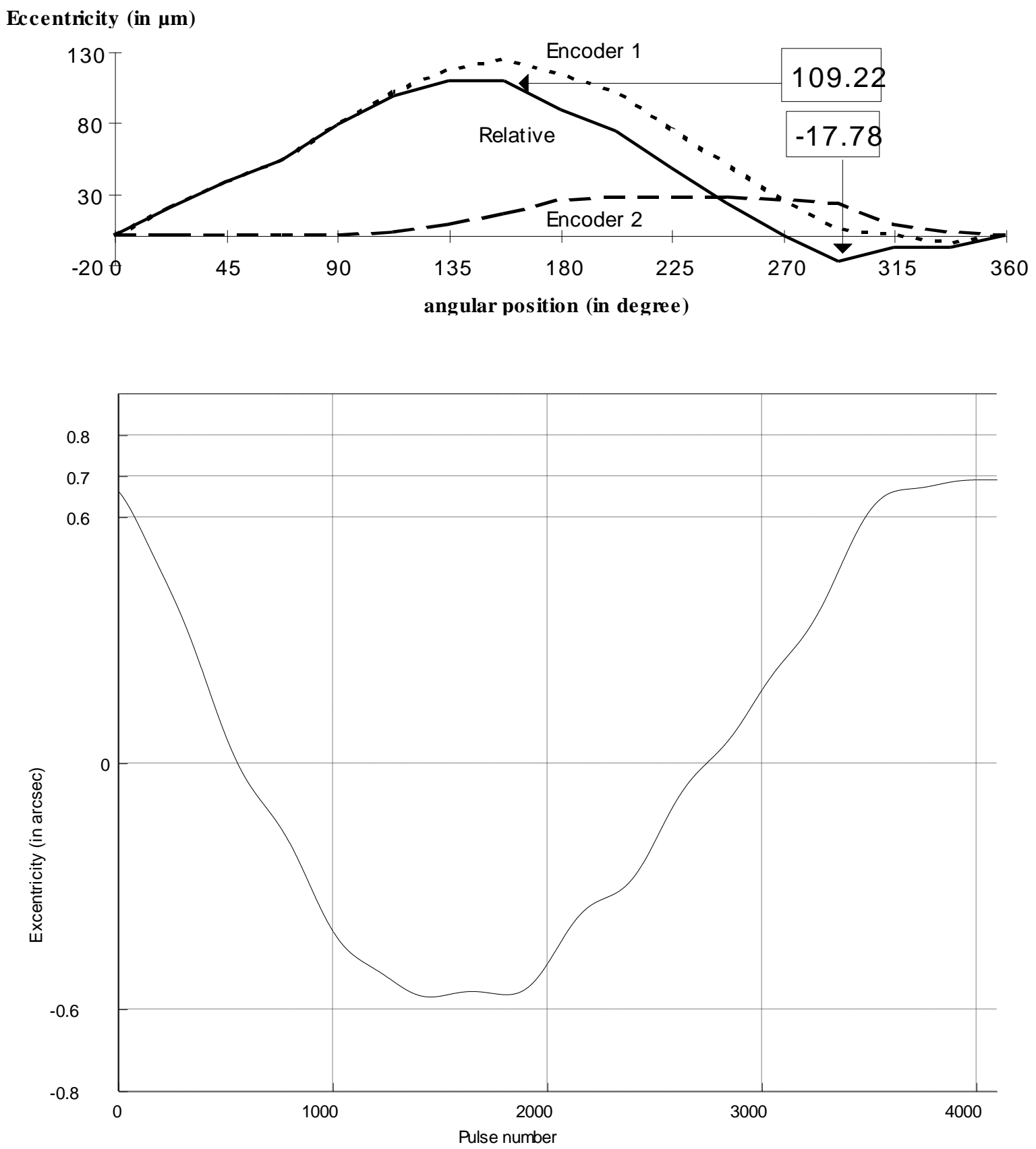

Fig. 7 : Comparison between two different measurements of relative eccentricity of encoder disks.

This measurement of relative eccentricity leads to an overall amplitude of 127 $\mu \mathrm{m}$ for an operating radius of cells of about $30 \mathrm{~mm}$. From equation (5), it can be estimated that the overall eccentricity measured with optical encoder should be of 1.34 arcsec, giving a difference of about $3 \%$ of the measured eccentricity by optical encoders. 


\section{Conclusions.}

An improved technique for measuring TE in high speed gear transmission with low pulse per revolution optical encoder is examined on its effective performances. This technique provides us with the opportunity to measure torsional vibrations or transmission error with a highly precise tool. Length of encoder pulses are estimated with a high frequency timer $(100 \mathrm{MHz})$ (we postulated that the speed stays constant during an encoder pulse and this is true for the range of precision required for T.E measurement at high speed). The theoretical precision of this device is shown to be dependent on only the angular speed of shaft, but not on the number of pulse per revolution of the encoder. The number of pulses per revolution of the encoder specifies the resolution, while intrinsic encoder accuracy (i.e. grating location or electronic signal processing) affects measurement precision. However, it has to be kept in mind that encoders with higher number of gratings are more accurate : thus, precision can be improved by using higher number of grating and then, by dividing the generated pulse signal. Obviously, this number of pulses per revolution has to match with number of gear teeth for proper gear harmonic analysis.

We showed that the determination of corrective data assigned to each gratings from a calibration set-up leads to a not significant improvement of the precision measurement. This fact is certainly induced by the mechanical behaviour of bearings, belts and pulleys, and motor. The coherence from one revolution to another does not reveal significant components. The overall precision achieved is less than about 0.03 second of arc on each frequency of the power spectral density. In order to prevent overestimation of eccentricity induced by distorded mounting of optical disks on shaft, it is possible to calibrate this component by measuring disk 
eccentricities with dial gauges. The correlation between the two measurements is less than $3 \%$ of the magnitude of relative eccentricity.

\section{Acknowledgements.}

The author would like to thank Professor Claude GOSSELIN for his welcoming support and his kind advises for this work during a visiting period in Mechanical Engineering Department, LAVAL University, Québec. The author is greatly indebted to the technical staff of the Department for their helps and meticulous works, in particular to Yves JEAN for his advises and assistance. 


\section{References.}

(1) C. BARD, D. REMOND and D. PLAY, "New Transmission Error Measurement for Heavy Load Gears", 1994 International Gearing Conference, 7-9 September 1994, Newcastle, England.

(2) C. BARD, D. REMOND et D. PLAY, "Une technique originale de mesure de l'erreur de transmission dans des conditions réelles de fonctionnement", Journées d'information "des engrenages plus silencieux", CETIM, Senlis, 21-22 oct. 1993, 6p.

(3) HOUSER D. R., BLANKENSHIP G. W., "Methods for measuring transmission error under load and at operating speeds", SAE Transactions, 1989, 98(6), 1367-1374.

(4) KONG X. "Gear train monitoring by transmission error method", Ph. D. Thesis : Mechanical Engineering, University of Wisconsin-Madison, 1987.

(5) MUNRO R. G., "A review of the theory and measurement of gear transmission error", Proc. of the Institution of Mechanical Engineers, first Int. Conf. gearbox noise and vibration, 9-11 April 1990, Unv. of Cambridge.

(6) REMOND D. "Contribution à l'étude et l'analyse expérimentale du bruit d'engrènement", Thèse de doctorat, Institut National des Sciences Appliquées de Lyon, 1991.

(7) REMOND D., VELEX P., SABOT J, "Comportement dynamique et acoustique des transmissions par engrenages, synthèse bibliographique", Publication CETIM,1993.

(8) SMITH J.D., "Gear transmission error accuracy with small rotary encoders", Proc. Instn Mech. Engrs, Part C, 1987, 201(C2), 133-135 
(9) SMITH J.D., "Identification of small amplitude resonances in rotary digital systems", Proc. Instn Mech. Engrs, 1988, 202(C1), 63-65.

(10) SMITH J. D, "A modular system for transmission error measurement", Proc. of the Institution of Mechanical Engineers, 1988, 202(C6), 439-442.

(11) SMITH J. D., "Comparing encoder and accelerometer measurement of transmission error or torsional vibration", Proc. of the Institution of Mechanical Engineers, first Int. Conf. gearbox noise and vibration, 9-11 April 1990, Unv. of Cambridge.

(12) SMITH J. D., "Practical rotary encoder accuracy limits for transmission error measurement", Proc. of the Instn Mech. Engrs, Part C, 1991, 205(C6), 431436.

(13) SMITH J.D.,"Alias errors in precision rotary encoder calibration", Proc. Instn Mech. Engrs, 1992, 206(C1), 71-73.

(14) SWEENEY P.J., RANDALL R.B., "Gear transmission error measurement using phase demodulation", Proc. Instn Mech. Engrs, Part C, 1996, 210(3), 201-213. 